\title{
On the concentration-compactness phenomenon for the first Schrodinger eigenvalue
}

\author{
Gerasim Kokarev \\ School of Mathematics, The University of Edinburgh \\ King's Buildings, Mayfield Road, Edinburgh EH9 3JZ, UK \\ Email: G.Kokarev@ed.ac.uk
}

\begin{abstract}
We study a variational problem for the first eigenvalue $\lambda_{1}(V)$ of the Schrodinger operator $\left(-\Delta_{g}+V\right)$ on closed Riemannian surfaces. More precisely, we explore concentration-compactness properties of sequences formed by $\lambda_{1}$-extremal potentials.
\end{abstract}

Mathematics Subject Classification (2000): 58C40, 58E30.

\section{Introduction}

Let $M$ be a closed smooth surface endowed with a Riemannian metric $g$ of volume one. For a function $V \in L^{\infty}(M)$ we denote by

$$
\lambda_{0}(V)<\lambda_{1}(V) \leqslant \ldots \leqslant \lambda_{k}(V) \leqslant \ldots
$$

eigenvalues of the Schrodinger operator $\left(-\Delta_{g}+V\right)$. We suppose that potentials $V$ have zero mean-values and denote their space by

$$
L_{0}^{\infty}(M)=\left\{V \in L^{\infty}(M): \int_{M} V d V o l_{g}=0\right\} .
$$

It is a simple exercise to see that the zero eigenvalue $\lambda_{0}(V)$ is non-positive for any $V \in L_{0}^{\infty}(M)$. The next eigenvalue $\lambda_{1}(V)$ is also bounded from above when $V$ ranges over $L_{0}^{\infty}(M)$. More precisely, by the work of $\mathrm{Li}$ and Yau [10], see also [3], the first eigenvalue $\lambda_{1}(V)$ can be estimated by the conformal volume; the latter can be bounded in terms of the genus $\gamma$ of $M$ in many cases. For example, for an orientable surface $M$ we have

$$
\lambda_{1}(V) \leqslant 8 \pi(\gamma+1),
$$

where $V$ ranges over $L_{0}^{\infty}(M)$.

We regard the first eigenvalue $\lambda_{1}(V)$ as a functional on the space of potentials $L_{0}^{\infty}(M)$, and are interested in its critical points. Following Nadirashvili [11], a potential $V$ is called $\lambda_{1}$-extremal if for any $q \in L_{0}^{\infty}(M)$ the function $\lambda_{1}(V+t q)$, where $t$ ranges in a neighbourhood of zero, satisfies either the inequality

$$
\lambda_{1}(V+t q) \leqslant \lambda_{1}(V)+o(t) \quad \text { as } \quad t \rightarrow 0,
$$

or the inequality

$$
\lambda_{1}(V+t q) \geqslant \lambda_{1}(V)+o(t) \quad \text { as } \quad t \rightarrow 0 .
$$


In particular, we see that any $\lambda_{1}$-maximal potential is extremal. Basic properties of extremal potentials are discussed in Sect. 1 Here we mention only that any extremal potential $V$ is $C^{\infty}$-smooth.

The purpose of this note is to communicate the following concentration-compactness alternative for the first eigenvalue functional.

Theorem A. Let $M$ be a closed surface endowed with a Riemannian metric $g$ of volume one and $V_{n} \in L_{0}^{\infty}(M), n=1,2, \ldots$, be a sequence of $\lambda_{1}$-extremal potentials for the Schrodinger operator $\left(-\Delta_{g}+V\right)$. Then there exists a subsequence $V_{n_{k}}$ such that one of the following holds:

(i) the subsequence $\left(V_{n_{k}}\right)$ converges in the sense of distributions to $8 \pi\left(1-\delta_{x}\right)$, for some $x \in M$, and $\lambda_{1}\left(V_{n_{k}}\right) \rightarrow 8 \pi$ as $n_{k} \rightarrow+\infty$;

(ii) the subsequence $\left(V_{n_{k}}\right)$ converges in $C^{\infty}$-topology to an extremal potential $V \in L_{0}^{\infty}(M)$, and $\lambda_{1}\left(V_{n_{k}}\right) \rightarrow \lambda_{1}(V)>0$ as $n_{k} \rightarrow+\infty$.

As a consequence, we see that the set of extremal potentials whose first eigenvalues are bounded away from $8 \pi$ is always compact. The critical value $8 \pi$ is the maximal first eigenvalue on the 2-sphere endowed with the standard round metric of volume one, and by Prop. 3 below maximal potentials on it form a non-compact space.

The proof of the alternative is based on the two ingredients: characterisation of extremal potentials via harmonic maps and the use of the bubbling convergence theorem for the latter. The proof that the critical value for the concentration can be only $8 \pi$ involves more detailed study of the Schrodinger equation near the bubble point. In the process we obtain a general upper estimate (Lemma4) for the critical value of a sequence of (not necessarily extremal!) concentrating potentials. Our methods, combined with the version of the bubbling convergence theorem in [12], also yield a version of the result for the case of a variable metric on $M$. We state it explicitly for the completeness.

Theorem B. Let $g_{n}, n=1,2, \ldots$, be a sequence of unit volume metrics on $M$ converging in $C^{2}$-topology to a metric $g$, and $V_{n}, n=1,2, \ldots$, be a sequence of potentials such that each $V_{n}$ is $\lambda_{1}$-extremal for the Schrodinger operator $\left(-\Delta_{g_{n}}+V\right)$. Then there exists a subsequence $\left(V_{n_{k}}\right)$ such that one of the following holds:

(i) the subsequence $\left(V_{n_{k}}\right)$ converges in the sense of distributions to $8 \pi\left(1-\delta_{x}\right)$, for some $x \in M$, and $\lambda_{1}\left(V_{n_{k}}\right) \rightarrow 8 \pi$ as $n_{k} \rightarrow+\infty$;

(ii) the subsequence $\left(V_{n_{k}}\right)$ converges in $C^{\infty}$-topology to an extremal potential $V \in L_{0}^{\infty}(M)$, and $\lambda_{1}\left(V_{n_{k}}\right) \rightarrow \lambda_{1}(V)>0$ as $n_{k} \rightarrow+\infty$.

In a forthcoming paper we shall study questions related to this concentration-compactness alternative in dimension greater than two.

Acknowledgments. The author is grateful to Nikolai Nadirashvili for a number of discussions on the subject. The author acknowledges the support of EPSRC and the Maxwell Mathematical Institute during the work on the paper. 


\section{Preliminaries}

\subsection{Notation. Properties of extremal potentials}

Let $M$ be a closed smooth surface. For a Riemannian metric $g$ on $M$ the Laplace operator $\Delta_{g}$ in local coordinates $\left(x^{i}\right), 1 \leqslant i \leqslant 2$, has the form

$$
\Delta_{g}=\frac{1}{\sqrt{|g|}} \frac{\partial}{\partial x^{i}}\left(\sqrt{|g|} g^{i j} \frac{\partial}{\partial x^{j}}\right),
$$

where $\left(g_{i j}\right)$ are components of the metric $g,\left(g^{i j}\right)$ is the inverse tensor, and $|g|$ stands for $\operatorname{det}\left(g_{i j}\right)$. We use above the summation convention for the repeated indices. We suppose throughout that $g$ is normalised such that $\operatorname{Vol}_{g}(M)$ equals one. For a function $V \in L_{0}^{\infty}(M)$ we denote by

$$
\lambda_{0}(V)<\lambda_{1}(V) \leqslant \ldots \leqslant \lambda_{k}(V) \leqslant \ldots
$$

the eigenvalues of the Schrodinger operator $\left(-\Delta_{g}+V\right)$; these are real numbers for which the equation

$$
\left(-\Delta_{g}+V\right) u=\lambda_{k} u
$$

has a non-trivial solution. The solutions of equation (1.1) are called eigenfunctions. Recall that by variational characterisation the eigenvalue $\lambda_{k}(V)$ is the infimum of the Rayleigh quotient

$$
\mathrm{R}_{V}(u)=\frac{\int_{M}|\nabla u|^{2} d V o l_{g}+\int_{M} V u^{2} d V o l_{g}}{\int_{M} u^{2} d V o l_{g}}
$$

over the set of all smooth functions $u$ that are $L_{2}$-orthogonal to the eigenfunctions for $\lambda_{0}$, $\lambda_{1}, \ldots, \lambda_{k-1}$. In particular, we see that

$$
\lambda_{0}(V) \leqslant \mathrm{R}_{V}(1)=0 \quad \text { for any } V \in L_{0}^{\infty}(M) .
$$

Now we discuss the properties of extremal potentials. The following proposition is a sharpened version of the one due to Nadirashvili [11]; below we outline the proof based on the first variation formula for $\lambda_{1}$.

Proposition 1. For a function $V \in L_{0}^{\infty}(M)$ the following hypotheses are equivalent:

(i) $V$ is a $\lambda_{1}$-extremal potential for the Schrodinger operator;

(ii) the quadratic form

$$
u \longmapsto \int_{M} q u^{2} d \operatorname{Vol}_{g}
$$

is indefinite on the space of $\lambda_{1}$-eigenfunctions of the Schrodinger operator $\left(-\Delta_{g}+V\right)$ for any $q \in L_{0}^{\infty}(M)$;

(iii) there exists a finite collection of $\lambda_{1}$-eigenfunctions $\left(u_{i}\right)$ of the Schrodinger operator $\left(-\Delta_{g}+V\right)$ such that $\sum_{i} u_{i}^{2}=1$.

Outline of the proof. Denote by $V_{t}$ the family of functions $(V+t q)$ in $L_{0}^{\infty}(M)$; we assume that $t$ ranges in a neighbourhood of zero. Suppose that the first eigenvalue $\lambda_{1}(V)$ has multiplicity $m$. Then by general perturbation theory [8] there exists $m$ analytic families of real numbers $\Lambda_{i, t}$ and functions $u_{i, t}$, where $i=1, \ldots, m$, such that

$$
\left(-\Delta_{g}+V_{t}\right) u_{i, t}=\Lambda_{i, t} u_{i, t}, \quad \Lambda_{i, 0}=\lambda_{1}(V) .
$$


Assume that the $L_{2}$-norms of the $u_{i, t}$ 's are equal to one. Differentiating relation (1.3) with respect to $t$ and evaluating the result at $t=0$, we obtain

$$
q u_{i, 0}+\left(-\Delta_{g}+V\right) u_{i, 0}^{\prime}=\Lambda_{i, 0}^{\prime} u_{i, 0}+\Lambda_{i, 0} u_{i, 0}^{\prime} .
$$

Multiplying the identities (1.3) and (1.4) by $u_{i, 0}^{\prime}$ and $u_{i, 0}$ respectively and substracting the first from the second, after integration, we obtain the first variation formula

$$
\left.\frac{d}{d t}\right|_{t=0} \Lambda_{i, t}=\int_{M} q u_{i, 0}^{2} d V l_{g} .
$$

The discussion implies that the function $\lambda_{1}\left(V_{t}\right)$ is piece-wise smooth and has left and right derivatives. Moreover, there exist indices $i$ and $j$ such that

$$
\left.\frac{d}{d t}\right|_{t=0-} \lambda_{1}\left(V_{t}\right)=\Lambda_{i, 0}^{\prime} \quad \text { and }\left.\quad \frac{d}{d t}\right|_{t=0+} \lambda_{1}\left(V_{t}\right)=\Lambda_{j, 0}^{\prime} .
$$

To prove the statement $(i) \Rightarrow(i i)$, we note that the potential $V$ is extremal if and only if

$$
\left.\left.\frac{d}{d t}\right|_{t=0-} \lambda_{1}\left(V_{t}\right) \cdot \frac{d}{d t}\right|_{t=0+} \lambda_{1}\left(V_{t}\right) \leqslant 0
$$

This together with relations above and formula (1.5) proves that the form (1.2) is indefinite.

To prove the converse statement $(i i) \Rightarrow(i)$ we note that in the basis $\left(u_{i, 0}\right)$, where $i=1, \ldots, m$, the quadratic form 1.2 is diagonal:

$$
\int_{M} q u_{i, 0} u_{j, 0} d \operatorname{Vol}_{g}=\left.\frac{d}{d t}\right|_{t=0} \Lambda_{i, t} \cdot \delta_{i j}
$$

This follows by differentiating relation (1.3) in the manner similar to the one used to obtain (1.5). Since $\lambda_{1}\left(V_{t}\right)$ equals $\min _{i} \Lambda_{i, t}$, we get

$$
\begin{gathered}
\left.\frac{d}{d t}\right|_{t=0+} \lambda_{1}\left(V_{t}\right)=\min _{i} \Lambda_{i, 0}^{\prime}=\min _{i} \int_{M} q u_{i, 0}^{2} d V o l_{g}, \\
\left.\frac{d}{d t}\right|_{t=0-} \lambda_{1}\left(V_{t}\right)=\max _{i} \Lambda_{i, 0}^{\prime}=\max _{i} \int_{M} q u_{i, 0}^{2} d V o l_{g} .
\end{gathered}
$$

Since the form (1.2) is indefinite, then either one of this derivatives vanishes or they have different signs. This means that the potential $V$ is extremal.

$(i i) \Rightarrow(i i i)$. Let $K$ be the convex hull of the set of squared $\lambda_{1}$-functions $\left\{u^{2}: u\right.$ is an eigenfunction $\}$. Suppose the contrary to the hypotheses $(i i i)$; then $1 \neq K$. By classical separation results, there exists a function $f \in L_{2}(M)$ such that

$$
\int_{M} 1 \cdot f d \operatorname{Vol}_{g}<0 \quad \text { and } \quad \int_{M} \phi \cdot f d V o l_{g}>0, \quad \text { where } \phi \in K \backslash\{0\} .
$$

Let $f_{0}$ be the mean-value part of $f$,

$$
f_{0}=f-\int_{M} f d \operatorname{Vol}_{g}
$$

Then for any eigenfunction $u$ we have

$$
\int_{M} f_{0} u^{2} d \operatorname{Vol}_{g}=\int_{M} f u^{2} d \operatorname{Vol}_{g}-\left(\int_{M} f d \operatorname{Vol}_{g}\right)\left(\int_{M} u^{2} d \operatorname{Vol}_{g}\right)>0 .
$$


This is a contradiction with $(i i)$.

(iii) $\Rightarrow($ ii $)$. Conversely, let $\left(u_{i}\right)$ be a finite collection of eigenfunctions satisfying the hypothesis (iii). Then for any $q \in L_{0}^{\infty}(M)$, we have

$$
\int_{M} q\left(\sum_{i} u_{i}^{2}\right) d \operatorname{Vol}_{g}=\int_{M} q d \operatorname{Vol}_{g}=0 .
$$

This demonstrates the hypothesis (ii).

As a consequence we point out the following properties of extremal potentials.

Corollary 2. Let $V \in L_{0}^{\infty}(M)$ be an extremal potential for the Schrodinger operator. Then $V$ is $C^{\infty}$-smooth, and is bounded by its first eigenvalue $\lambda_{1}(V) \geqslant V$. Besides, the equality above occurs at most at a finite number of points, and the first eigenvalue is positive.

Proof. Since $V$ is extremal, by the proposition above there exists a collection of eigenfunctions $\left(u_{i}\right), i=1, \ldots, k$, such that $\sum_{i} u_{i}^{2}=1$. This means that the map

$$
M \ni x \longmapsto\left(u_{1}(x), \ldots, u_{k}(x)\right) \in S^{k-1} \subset \mathbf{R}^{k}
$$

is weakly harmonic, see [5]. Since the dimension of $M$ equals 2, the eigenfunctions are continuous, and by standard regularity theory [9] the map given by (1.6) is actually $C^{\infty}$ smooth. Applying the Laplacian to the identity $\sum_{i} u_{i}^{2}=1$, we further obtain the relation

$$
V=\lambda_{1}(V)-\sum_{i}\left|\nabla u_{i}\right|^{2} .
$$

Thus, the potential $V$ is also $C^{\infty}$-smooth, and is bounded by its first eigenvalue. The points where $\lambda_{1}(V)$ equals $V$ corresond to the branch points of the harmonic map (1.6); there can be only finite number of these unless the harmonic map is constant. The latter can not occur. For otherwise, relation (1.7) together with the hypothesis $V \in L_{0}^{\infty}(M)$ imply that both $V$ and $\lambda_{1}(V)$ vanish identically. Thus, $\lambda_{1}(V)$ becomes the first eigenvalue of the Laplacian $\left(-\Delta_{g}\right)$, which is strictly positive - a contradiction. Finally, the positivity of $\lambda_{1}(V)$ follows by integration of (1.7).

In sequel we freely identify collections of eigenfunctions $\left(u_{i}\right)$ such that $\sum_{i} u_{i}^{2}=1$ with harmonic maps into round spheres. For basic properties and facts on the latter we refer to excellent texts [2, 5].

\subsection{Examples of extremal potentials}

Here we mention simplest examples of $\lambda_{1}$-extremal potentials. We start with the case when $M$ is a standard round sphere. The following proposition is a version of the theorem of Hersch [6].

Proposition 3. Let $M$ be a 2-sphere endowed with the standard round metric $g$ of volume one. Then the maximal first eigenvalue of the Schrodinger operator is equal to $8 \pi$, and is achieved by the zero potential. Further, any extremal potential on $M$ is maximal, and has the form

$$
V(x)=64 \pi^{2}-8 \pi|\nabla s|^{2}(x), \quad x \in M,
$$

where $s: S^{2} \rightarrow S^{2}$ is a Mobious transformation and $|\nabla s|$ stands for the Hilbert-Schmidt norm of its differential. In particular, the space of maximal potentials is non-compact.

The proof is outlined below; the key ingredient is the following lemma, see [6, 10]. 
Hersch's Lemma. Let $y^{i}, i=1,2,3$, be coordinate functions in $\mathbf{R}^{3}$, and $\phi: M \rightarrow S^{2} \subset \mathbf{R}^{3}$ be a conformal map to the unit sphere centred at the origin. Then for any absolutely continuous measure $\mu$ on $M$ there exists a conformal diffeomorphism $s: S^{2} \rightarrow S^{2}$ such that

$$
\int_{M}\left(y^{i} \circ s \circ \phi\right) d \mu=0, \quad \text { for all } i=1,2,3 .
$$

Proof of Prop. 3 First, we show that the zero potential is maximal. Its first eigenvalue is the first Laplacian eigenvalue of the standard round metric of volume one, and is equal to $8 \pi$. By Hersch's lemma for any $V \in L_{0}^{\infty}(M)$ the exists a conformal diffeomorphism $s: S^{2} \rightarrow S^{2}$ such that the functions $\left(y^{i} \circ s\right), i=1,2,3$, are $L_{2}$-orthogonal to the ground state of the Schrodinger operator $\left(-\Delta_{g}+V\right)$. Thus, by variational characterisation, we have

$$
\lambda_{1}(V) \int_{M}\left(y^{i} \circ s\right)^{2} d V o l_{g} \leqslant \int_{M}\left|\nabla\left(y^{i} \circ s\right)\right|^{2} d V^{\prime} l_{g}+\int_{M} V\left(y^{i} \circ s\right)^{2} d V o l_{g}
$$

for any $i=1,2,3$. Since the volume of $g$ equals one, summing these identities, we obtain

$$
\lambda_{1}(V) \leqslant \sum_{i} \int_{M}\left|\nabla\left(y^{i} \circ s\right)\right|^{2} d \operatorname{Vol}_{g}=\sum_{i} \int_{M}\left|\nabla y^{i}\right|^{2} d V o l_{g}=8 \pi
$$

here the first equality holds by the conformal invariance of the Dirichlet energy. Thus, $\lambda_{1}(V) \leqslant 8 \pi$, and the zero potential is, indeed, maximal.

Now we show that any extremal potential $V$ is, in fact, maximal. By Prop. 1 there exists a collection of first eigenfunctions $\left(u_{i}\right), i=1, \ldots, k$, such that $\sum_{i} u_{i}^{2}=1$. By Cor. 2 the potential $V$ is $C^{\infty}$-smooth, and the result in [1] says that the multiplicity of its first eigenvalue is not greater than 3. Thus, the harmonic map (1.6), defined by eigenfunctions $\left(u_{i}\right)$, lies in the section of the unit sphere by a subspace whose dimension is not greater than 3. In other words, this harmonic map is a map into the 2-dimensional unit sphere. As is known [2], its energy

$$
\int_{M} \sum_{i}\left|\nabla u_{i}\right|^{2} d \operatorname{Vol}_{g}
$$

is an integer multiple of $8 \pi$. By relation (1.7), it coincides with $\lambda_{1}(V)$, and by the discussion above can be either zero or $8 \pi$. By Cor. 2, the former can not occur. Thus, the first eigenvalue $\lambda_{1}(V)$ equals to $8 \pi$, and the potential $V$ is maximal.

Finally, [3, Cor 2.7] implies that any maximal potential $V$ on the standard 2-sphere has the form

$$
V=64 \pi^{2}-8 \pi \sum_{i}\left|\nabla\left(y^{i} \circ s\right)\right|^{2}
$$

where $s$ is a Mobious transformation of $S^{2}$. The latter form a non-compact group $\operatorname{PSL}(2, \mathbf{C})$, and the space of maximal potentials can be identified with its topological quotient by the equivalence relation

$$
s_{1} \sim s_{2} \quad \text { iff } \quad\left|\nabla s_{1}\right|^{2}=\left|\nabla s_{2}\right|^{2} .
$$

It is a straightforward calculation to see that the natural projection onto $\operatorname{PSL}(2, \mathbf{C}) \backslash \sim$ is proper and, in particular, the quotient $\operatorname{PSL}(2, \mathbf{C}) \backslash \sim$ has to be non-compact.

It is also straightforward to construct examples of extremal potentials on tori. For example, by Prop. 1 for any flat torus the zero potential is extremal for the first eigenvalue. Moreover, if $M$ is the Clifford torus (the quotient by the lattice $\mathbf{Z}(1,0) \oplus \mathbf{Z}(0,1)$ ) or the equilateral torus (the quotient by $\mathbf{Z}(1,0) \oplus \mathbf{Z}(1 / 2, \sqrt{3} / 2)$ ), then the zero potential is a unique global maximiser in $L_{0}^{\infty}(M)$; see [3, 4] for the details. 


\section{Proof of Theorem A: the alternative}

\subsection{The setup}

Let $V_{n}, n=1,2, \ldots$, be a given sequence of extremal potentials. Since the $\lambda_{1}\left(V_{n}\right)$ 's are nonnegative and, by [3, 10], uniformly bounded, without loss of generality we can suppose that the sequence $\lambda_{1}\left(V_{n}\right)$ converges to a limit $\lambda_{*} \geqslant 0$. By Prop. 1 for each $n \in \mathbf{N}$ there exists a finite collection of eigenfunctions $\left(u_{i, n}\right), i=1, \ldots, m_{n}$, such that $\sum_{i} u_{i, n}^{2}=1$. Since the potentials $V_{n}$ 's are smooth, by the results in [1] the multiplicities of the $\lambda_{1}\left(V_{n}\right)$ 's are uniformly bounded in terms of the genus of $M$ only. Thus, after a selection of a subsequence, we may suppose that for each $n \in \mathbf{N}$ there exists the same number of eigenfunctions $\left(u_{i, n}\right)$, $i=1, \ldots, m$, such that $\sum_{i} u_{i, n}^{2}=1$. In other words, for each potential $V_{n}$ we have a harmonic map

$$
M \ni x \longmapsto U_{n}(x)=\left(u_{i, n}(x)\right) \in S^{m-1} \subset \mathbf{R}^{m} .
$$

As in the proof of Corollary 2 , we see that

$$
\left|\nabla U_{n}\right|^{2}=\sum_{i}\left|\nabla u_{i, n}\right|^{2}=\lambda_{1}\left(V_{n}\right)-V_{n} .
$$

In particular, the energies

$$
E\left(U_{n}\right):=\int_{M}\left|\nabla U_{n}\right|^{2} d V o l_{g}
$$

of these harmonic maps are equal to $\lambda_{1}\left(V_{n}\right)$ and, hence, are bounded. Now by the bubbling convergence theorem for harmonic maps [13, 7], there exists a subsequence, also denoted by $\left(U_{n}\right)$, which converges weakly in $W^{1,2}\left(M, S^{m-1}\right)$ to a harmonic map $U: M \rightarrow S^{m-1}$. Moreover, there exists a finite number of "bubble points" $\left\{x_{1}, \ldots, x_{\ell}\right\} \subset M$ such that the $U_{n}$ 's converge in $C^{\infty}$-topology on compact sets in $M \backslash\left\{x_{1}, \ldots, x_{\ell}\right\}$, and the energy densities $\left|\nabla U_{n}\right|^{2}$ converge weakly in the sense of measures to $|\nabla U|^{2}$ plus a finite sum of Dirac measures:

$$
\left|\nabla U_{n}\right|^{2} \rightarrow|\nabla U|^{2}+\sum_{j} e_{j} \delta_{x_{j}}
$$

where the constants $e_{j}>0$ correspond to the energies of the so-called bubble spheres, see Sect.3. 4.

Now we consider two cases when the energy density $|\nabla U|^{2}$ of the limit map vanishes identically or not. In the former case we obtain the claim $(i)$; the case when $|\nabla U|^{2} \not \equiv 0$ corresponds to the claim (ii).

\subsection{The case $|\nabla U|^{2} \equiv 0$ : concentration to a single point}

First, we show that there is at least one "bubble point". For otherwise, the harmonic maps $U_{n}$ converge in $C^{\infty}$-topology to a constant map $U$. By relation (2.1) we then conclude that the potentials $V_{n}$ converge in $C^{\infty}$-topology to zero and so do their first eigenvalues $\lambda_{1}\left(V_{n}\right)$. The latter implies that the Laplacian $\left(-\Delta_{g}\right)$ has constant first eigenfunctions - a contradiction. Thus, the energy measures of the harmonic maps $U_{n}$ converge weakly to a sum of Dirac-measures,

$$
\left|\nabla U_{n}\right|^{2}-\mu=\sum_{j} e_{j} \delta_{x_{j}} .
$$

Now we show that at most one delta-function can occur in the sum above.

Suppose the contrary. Then there are at least two "bubble points" $x_{1}$ and $x_{2}$. Denote by $\Omega_{1}$ and $\Omega_{2}$ their open non-intersecting coordinate neighbourhoods that do contain any other 
"bubble points". Since a point has zero capacity, then for any $\varepsilon>0$ there exist functions $\varphi_{i} \in C_{0}^{\infty}\left(\Omega_{i}\right)$ such that $0 \leqslant \varphi_{i} \leqslant 1$,

$$
\varphi_{i}=1 \text { in a neighbourhood of } x_{i}, \quad \text { and } \int_{M}\left|\nabla \varphi_{i}\right|^{2} d V o l_{g}<\varepsilon, \quad i=1,2 .
$$

Let $v_{n}$ be a $\lambda_{0}$-eigenfunction (ground state) of the Schrodinger operator $\left(-\Delta_{g}+V_{n}\right)$. Further, let $\alpha_{1, n}$ and $\alpha_{2, n}$ be two sequences of real numbers such that the linear combinations $\sum_{i} \alpha_{i, n} \varphi_{i}$ are $L_{2}$-orthogonal to the $v_{n}$ 's, and the sum of squares $\sum_{i} \alpha_{i}^{2}$ equals one for any $n$. Without loss of generality, we may suppose that the $\alpha_{i, n}$ 's converge to some $\alpha_{i}$ 's; the limit $\alpha_{i}$ 's clearly satisfy the relation $\sum_{i} \alpha_{i}^{2}=1$. Finally, denote by $\psi_{n}$ and $\psi$ the functions $\sum_{i} \alpha_{i, n} \varphi_{i}$ and $\sum_{i} \alpha_{i} \varphi_{i}$ respectively.

By construction, each function $\psi_{n}$ is $L_{2}$-orthogonal to $v_{n}$, and by variational principle we have

$$
\lambda_{1}\left(V_{n}\right) \int_{M} \psi_{n}^{2} d \operatorname{Vol}_{g} \leqslant \int_{M}\left|\nabla \psi_{n}\right|^{2} d \operatorname{Vol}_{g}+\int_{M} V_{n} \psi_{n}^{2} d V o l_{g} .
$$

Since $V_{n} \rightarrow \lambda_{*}-\sum_{j} e_{j} \delta_{x_{j}}$, then passing to the limit, we obtain

$$
\lambda_{*} \int_{M} \psi^{2} d \operatorname{Vol}_{g} \leqslant \int_{M}|\nabla \psi|^{2} d V o l_{g}+\lambda_{*} \int_{M} \psi^{2} d \operatorname{Vol}_{g}-\sum_{i} \alpha_{i}^{2} e_{i}
$$

The last relation implies

$$
\sum_{i} \alpha_{i}^{2} e_{i} \leqslant \int_{M}|\nabla \psi|^{2} d \operatorname{Vol}_{g} \leqslant \varepsilon
$$

Choosing $\varepsilon<\min \left\{e_{i}\right\}$, we obtain a contradiction. Thus, the limit measure $\mu$ is one-point supported; $\mu=e \delta_{x}$ for some $x \in M$.

Since the potentials $V_{n}$ 's have zero mean-value, we conclude from (2.1) that the constant $e$ equals $\lambda_{*}$. Now for a proof of the claim $(i)$ it remains to show that $\lambda_{*}$ equals $8 \pi$. We explain this in Sect. 3 .

\subsection{The case $|\nabla U|^{2} \not \equiv 0$ : regularity of the limit measure}

Recall that by the bubbling convergence theorem for harmonic maps, the energy densities $\left|\nabla U_{n}\right|^{2}$ converge weakly to the measure

$$
\mu=|\nabla U|^{2}+\sum_{j} e_{j} \delta_{x_{j}}
$$

here we suppose that $U: M \rightarrow S^{m-1}$ is a non-trivial harmonic map. First, the argument similar to the one in Sect. 2.2 shows that there is at most one "bubble point". More precisely, if we suppose the contrary, then for a given $\varepsilon>0$ we can choose the neighbourhoods $\Omega_{1}$ and $\Omega_{2}$ such that

$$
\sum_{i} \int_{\Omega_{i}}|\nabla U|^{2} d \operatorname{Vol}_{g}<\varepsilon
$$

The potentials $V_{n}$ converge weakly to $\lambda_{*}-\mu$, and passing to the limit in inequality (2.2), we obtain

$$
\sum_{i} \alpha_{i}^{2} e_{i} \leqslant \int_{M}|\nabla \psi|^{2} d \operatorname{Vol}_{g}+\sum_{i} \int_{\Omega_{i}}|\nabla U|^{2} d \text { Vol }_{g} \leqslant 2 \varepsilon .
$$

Now choosing $\varepsilon$ such that $2 \varepsilon<\min \left\{e_{i}\right\}$, we obtain a contradiction. Thus, the limit measure $\mu$ has the form $|\nabla U|^{2}+e \delta_{x}$. 
We claim that one "bubble point" can not occur also, and the limit measure is absolutely continuous. Suppose the contrary. Let $\Omega$ be a coordinate ball centred at the "bubble point" $x$. Since the capacity of a point is zero, then for any $\varepsilon>0$ there exists a function $\varphi \in C_{0}^{\infty}(\Omega)$ such that $0 \leqslant \varphi \leqslant 1$,

$$
\varphi=1 \text { in a neighbourhood of } x, \text { and } \int_{M}|\nabla \varphi|^{2} d V o l_{g}<\varepsilon .
$$

As in Sect. 2.2 by $v_{n}$ we denote positive ground states of the Schrodinger operators $\left(-\Delta_{g}+V_{n}\right)$; we assume that their $L_{1}$-norms are equal to one. Consider the sequence

$$
0<\alpha_{n}=\int_{M} \varphi \cdot v_{n} d V o l_{g} \leqslant 1
$$

without loss of generality, we may assume that the $\alpha_{n}$ 's converge to some limit $\alpha \geqslant 0$. By $\psi_{n}$ we denote the functions $\left(\varphi-\alpha_{n}\right)$, and by $\psi$ the function $(\varphi-\alpha)$. Since each $\psi_{n}$ is $L_{2}$-orthogonal to $v_{n}$, by variational principle we have

$$
\lambda_{1}\left(V_{n}\right) \int_{M} \psi_{n}^{2} d \operatorname{Vol}_{g} \leqslant \int_{M}\left|\nabla \psi_{n}\right|^{2} d V o l_{g}+\int_{M} V_{n} \psi_{n}^{2} d V_{o l} .
$$

Since $V_{n} \rightarrow \lambda_{*}-|\nabla U|^{2}-e \delta_{x}$, then passing to the limit and making elementary transformations, we obtain

$$
\int_{M}|\nabla U|^{2} \psi^{2} d \operatorname{Vol}_{g}+e(1-\alpha)^{2} \leqslant \int_{M}|\nabla \psi|^{2} d \operatorname{Vol}_{g} .
$$

The last relation implies

$$
\alpha^{2} \int_{M \backslash \Omega}|\nabla U|^{2} d \operatorname{Vol}_{g}+(1-\alpha)^{2} e \leqslant \int_{M}|\nabla \varphi|^{2} d \operatorname{Vol}_{g}<\varepsilon
$$

By elementary analysis, the left-hand side is bounded below by

$$
0<\left(e \int_{M \backslash \Omega}|\nabla U|^{2} d V o l_{g}\right) /\left(e+\int_{M \backslash \Omega}|\nabla U|^{2} d V o l_{g}\right) .
$$

This yields a contradiction, since $\varepsilon>0$ is arbitrary.

Thus, we see that no bubbling can occur, and the harmonic maps $U_{n}$ converge in $C^{\infty}$ topology to the harmonic map $U$. Further, by relation (2.1) the extremal potentials $V_{n}$ also converge in $C^{\infty}$-topology to the potential

$$
V=\lambda_{*}-|\nabla U|^{2}, \quad V \in L_{0}^{\infty}(M) .
$$

By standard perturbation theory [8], the eigenvalues $\lambda_{1}\left(V_{n}\right)$ has to converge to $\lambda_{1}(V)$, and we conclude that the first eigenvalue $\lambda_{1}(V)$ coincides with $\lambda_{*}$. Further, we see that the components $u_{i}, i=1, \ldots, m$, of the harmonic map $U$ are first eigenfunctions of the Schrodinger operator $\left(-\Delta_{g}+V\right)$. Finally, since $\sum_{i} u_{i}^{2}=1$, Prop. 1 implies that the potential $V$ is extremal.

\section{Proof of Theorem A: the eigenvalue of the bubble.}

For a proof of Theorem $\mathrm{A}$ it remains to show that the hypotheses $V_{n} \rightarrow \lambda_{*}\left(1-\delta_{x}\right)$, and $\lambda_{1}\left(V_{n}\right) \rightarrow \lambda_{*}$ as $n \rightarrow+\infty$ imply that $\lambda_{*}$ has to be equal to $8 \pi$. This is the content of the present section. First, we prove the estimate $\lambda_{*} \leqslant 8 \pi$ for concentrating sequences of not necessarily extremal potentials. To get the lower bound we study the Schrodinger equation on the bubble sphere obtained as the limit equation under convergence of renormalised eigenfunctions. 


\subsection{General upper bound: $\lambda_{*} \leqslant 8 \pi$}

The following lemma gives an estimate for arbitrary concentrating sequences of potentials; cf. [11, p.888-889].

Lemma 4. Let $M$ be a closed surface endowed with a Riemannian metric $g$, and $V_{n} \in L_{0}^{\infty}(M), n=1,2, \ldots$, be a sequence such that $V_{n} \rightarrow \lambda_{*}\left(1-\delta_{x}\right)$, and $\lambda_{1}\left(V_{n}\right) \rightarrow \lambda_{*}$ as $n \rightarrow+\infty$. Then the number $\lambda_{*}$ is not greater than $8 \pi$.

Proof. Let $\Omega$ be an open coordinate ball around $x \in M$ on which the metric $g$ is conformally Euclidean, and let

$$
\phi: \Omega \longrightarrow S^{2} \subset \mathbf{R}^{3}
$$

be a conformal map into the unit sphere in $\mathbf{R}^{3}$. Since a point on the Euclidean plane has zero capacity, then for any $\varepsilon>0$ there exists a function $\psi \in C_{0}^{\infty}(\Omega)$ such that $0 \leqslant \psi \leqslant 1$,

$$
\psi=1 \text { in a neighbourhood of } x, \text { and } \int_{M}|\nabla \psi|^{2} d \operatorname{Vol}_{g}<\varepsilon .
$$

As above by $v_{n}$ we denote a positive ground state of the Schrodinger operator $\left(-\Delta_{g}+V_{n}\right)$. By Hersch's lemma, Sect.1, there exists a conformal transformation $s_{n}: S^{2} \rightarrow S^{2}$ such that

$$
\int_{M} \psi\left(y^{i} \circ s_{n} \circ \phi\right) v_{n} d \operatorname{Vol}_{g}=0 \quad \text { for any } \quad i=1,2,3
$$

where $\left(y^{i}\right)$ are coordinate functions in $\mathbf{R}^{3}$. In other words, each function $\varphi_{n}^{i}=\psi\left(y^{i} \circ s_{n} \circ \phi\right)$ is $L_{2}$-orthogonal to $v_{n}$, and by variational principle we have

$$
\lambda_{1}\left(V_{n}\right) \int_{M}\left(\varphi_{n}^{i}\right)^{2} d V o l_{g} \leqslant \int_{M}\left|\nabla \varphi_{n}^{i}\right|^{2} d V o l_{g}+\int_{M} V_{n}\left(\varphi_{n}^{i}\right)^{2} d V o l_{g},
$$

for any $i=1,2,3$. Summing with respect to $i$, we obtain

$$
\lambda_{1}\left(V_{n}\right) \int_{M} \psi^{2} d \operatorname{Vol}_{g} \leqslant \sum_{i} \int_{M}\left|\nabla \varphi_{n}^{i}\right|^{2} d V o l_{g}+\int_{M} V_{n} \psi^{2} d V o l_{g}
$$

Now we estimate the first term on the right-hand side

$$
\begin{aligned}
\sum_{i} \int_{M}\left|\nabla \varphi_{n}^{i}\right|^{2} d V l_{g} \leqslant \sum_{i} \int_{M} & \psi^{2}\left|\nabla\left(y^{i} \circ s_{n} \circ \phi\right)\right|^{2} d \operatorname{Vol}_{g} \\
& +2 \sum_{i} \int_{M} \psi\left|\nabla\left(y^{i} \circ s_{n} \circ \phi\right)\right||\nabla \psi| d \operatorname{Vol}_{g}+\int_{M}|\nabla \psi|^{2} d V_{\text {Vol }} .
\end{aligned}
$$

The first sum on the right-hand side can be further estimated by the quantity

$$
\sum_{i} \int_{\Omega}\left|\nabla\left(y^{i} \circ s_{n} \circ \phi\right)\right|^{2} d \operatorname{Vol}_{g} \leqslant \sum_{i} \int_{S^{2}}\left|\nabla\left(y^{i} \circ s_{n}\right)\right|^{2} d V o l_{S^{2}}=8 \pi ;
$$

here we used the conformal invariance of the Dirichlet energy, which in particular implies that the energy of a conformal diffeomorphism of $S^{2}$ equals $8 \pi$. Similarly the second sum is not greater that

$$
\begin{aligned}
2 \sum_{i} \int_{\Omega}\left|\nabla\left(y^{i} \circ s_{n} \circ \phi\right)\right||\nabla \psi| d \operatorname{Vol}_{g} \leqslant 2 \varepsilon^{1 / 2} \sum_{i}\left(\int_{\Omega}\left|\nabla\left(y^{i} \circ s_{n} \circ \phi\right)\right|^{2} d V o l_{g}\right)^{1 / 2} & \\
& \leqslant 10 \pi^{1 / 2} \varepsilon^{1 / 2}
\end{aligned}
$$


Using these two estimates and the fact that the Dirichlet energy of $\psi$ is less than $\varepsilon$, we obtain

$$
\sum_{i} \int_{M}\left|\nabla \varphi_{n}^{i}\right|^{2} d V o l_{g} \leqslant 8 \pi+10 \pi^{1 / 2} \varepsilon^{1 / 2}+\varepsilon .
$$

Combining the last inequality with the one in $\underline{3.10}$, and passing to the limit as $n \rightarrow+\infty$, we arrive at the following relation

$$
\lambda_{*} \leqslant 8 \pi+10 \pi^{1 / 2} \varepsilon^{1 / 2}+\varepsilon .
$$

Since $\varepsilon>0$ is arbitrary, we conclude that $\lambda_{*} \leqslant 8 \pi$.

\subsection{The Schrodinger equation on the bubble sphere}

To obtain the lower bound $\lambda_{*} \geqslant 8 \pi$, we study a certain Schrodinger equation on the socalled bubble sphere. Bubble spheres appear as natural "limit objects" of sequences of renormalised harmonic maps, describing the behaviour of sequences near bubble points; see [13, 12]. The construction of a bubble sphere below uses a slightly non-standard renormalisation procedure that is more suitable in our context.

We start with a sequence of harmonic maps

$$
M \ni x \longmapsto U_{n}(x)=\left(u_{i, n}(x)\right) \in S^{m-1} \subset \mathbf{R}^{m}
$$

whose coordinates $u_{i, n}$ are first eigenfunctions of the Schrodinger operator $\left(-\Delta_{g}+V_{n}\right)$. We consider the case when the concentration occurs - the sequence $\left|\nabla U_{n}\right|^{2}$ converges weakly to the one-point supported singular measure $\lambda_{*} \delta_{x}$, see Sect. 2] In particular,

$$
\Lambda_{n}=\max _{x \in M}\left|\nabla U_{n}\right|^{2}(x) \rightarrow+\infty \quad \text { as } n \rightarrow+\infty .
$$

Let $x_{n} \in M$ be a point where the maximum of $\left|\nabla U_{n}\right|^{2}(x)$ is achieved; without loss of generality, we can assume that the $x_{n}$ 's converge to a point $x_{*} \in M$. Let $\Omega$ be a chart ball centred at $x_{*}$; we suppose that the metric $g$ is conformally Euclidean on $\Omega$ and $g_{i j}\left(x_{*}\right)=\delta_{i j}$. For a sufficiently large $n$, the mapping

$$
\phi_{n}: D_{n}=\left\{x \in \mathbf{R}^{2}:|x|<\sqrt{\Lambda_{n}}\right\} \rightarrow \Omega, \quad x \rightarrow x / \Lambda_{n}+x_{n},
$$

is well-defined. We endow the ball $D_{n}$ with a Riemannian metric $\left(g_{n}\right)_{i j}=g_{i j} \circ \phi_{n}$; equivalently, the $g_{n}$ equals $\Lambda_{n}^{2}\left(\phi_{n}^{*} g\right)$. Consider the functions $\bar{u}_{i, n}=u_{i, n} \circ \phi_{n}$ on each $D_{n}$; they satisfy the equations

$$
-\Delta_{g_{n}} \bar{u}_{i, n}=\frac{1}{\Lambda_{n}^{2}}\left(\lambda_{1}\left(V_{n}\right)-\bar{V}_{n}\right) \bar{u}_{i, n},
$$

where $\bar{V}_{n}=V_{n} \circ \phi_{n}$. Applying the Laplacian $\Delta_{g_{n}}$ to the identity $\sum_{i} \bar{u}_{i, n}^{2}=1$, we conclude that the maps

$$
D_{n} \ni x \longmapsto \bar{U}_{n}(x)=\left(\bar{u}_{i, n}(x)\right) \in S^{m-1} \subset \mathbf{R}^{m}
$$

are harmonic and satisfy the relations

$$
\left|\nabla \bar{U}_{n}\right|_{g_{n}}^{2}=\frac{1}{\Lambda_{n}^{2}}\left(\lambda_{1}\left(V_{n}\right)-\bar{V}_{n}\right) .
$$

By the definition of the $\phi_{n}$ 's, we also have

$$
\left|\nabla \bar{U}_{n}\right|_{g_{n}}(x) \leqslant 1, \quad \text { and } \quad\left|\nabla \bar{U}_{n}\right|_{g_{n}}(0)=1 .
$$


Since the metrics $g_{n}$ converge to the Euclidean metric on $\mathbf{R}^{2}$, the first inequality above together with standard Schauder estimates, see [9], imply that the maps $\bar{U}_{n}$ converge in $C^{\infty}$-topology to a harmonic map $\bar{U}: \mathbf{R}^{2} \rightarrow \Omega$ on each compact subset of $\mathbf{R}^{2}$. Finally, since the Dirichlet energy is conformally invariant, it is straightforward to show that

$$
\limsup \int_{D_{n}}\left|\nabla \bar{U}_{n}\right|_{g_{n}}^{2} d \operatorname{Vol}_{g_{n}} \leqslant \lambda_{*} \quad \text { as } n \rightarrow+\infty .
$$

Besides, if $x_{*}$ does not coincide with the bubble point $x$, the limsup on the left-hand side above vanishes.

Identifying $\mathbf{R}^{2}$ with $S^{2} \backslash\{p\}$ via the stereographic projection, we can view $\bar{U}=\left(\bar{u}_{i}\right)$, $i=1, \ldots, m$, as a harmonic map from $S^{2} \backslash\{p\} \rightarrow S^{m-1}$, where the sphere $S^{2}$ is endowed with the standard round metric $g_{s}$. Using the conformal invariance of energy again, we conclude from inequality (3.5) that the map $\bar{U}$ has finite energy, $E(\bar{U}) \leqslant \lambda_{*}$. Hence, by [13] its singularity at $p$ is removable - the map $\bar{U}$ extends to a smooth harmonic map $S^{2} \rightarrow S^{m-1}$. By the second relation in (3.4), the map $\bar{U}$ is non-constant, and its energy $E(\bar{U})$ is strictly positive. In particular, we conclude that the point $x_{*}$ coincides with the bubble point $x$. Denote the energy of $\bar{U}$ by $\bar{\lambda}$ and define the potential $\bar{V}$ on the sphere $S^{2}$ by the formula

$$
\bar{V}=\bar{\lambda}-|\nabla \bar{U}|_{g_{s}}^{2} .
$$

Clearly, it belongs to the space $L_{0}^{\infty}\left(S^{2}\right)$, and by relation (3.3) we have

$$
\frac{\kappa}{\Lambda_{n}^{2}}\left(\lambda_{1}\left(V_{n}\right)-\bar{V}_{n}\right) \rightarrow \bar{\lambda}-\bar{V}
$$

in $C^{\infty}$-topology on compact sets in $S^{2} \backslash\{p\}$. Here $\kappa$ stands for the conformal factor between the Euclidean metric on $S^{2} \backslash\{p\}$ and the standard metric $g_{s}$ on $S^{2}$. Since the Laplacian is conformally invariant in dimension two, then passing to the limit in equation (3.2), we obtain

$$
\left(-\Delta_{g_{s}}+\bar{V}\right) \bar{u}_{i}=\bar{\lambda} \bar{u}_{i}, \quad \text { where } i=1, \ldots, m .
$$

Thus, we see that $\bar{\lambda}$ is an eigenvalue for the Schrodinger operator $\left(-\Delta_{g_{s}}+\bar{V}\right)$ on the sphere, and the $\bar{u}_{i}$ 's are its eigenfunctions.

Lemma 5. The eigenfunctions $\bar{u}_{i}, i=1, \ldots, m$, span a vector space whose dimension is at most 3.

By Lemma 5 , we see that the harmonic map $\bar{U}$, defined by eigenfunctions $\left(\bar{u}_{i}\right)$, lies in the section of the unit sphere by a subspace whose dimension is not greater than 3 . In other words the harmonic map $\bar{U}$ is actually a harmonic map into the 2-dimensional unit sphere. Hence, its energy is an integer multiple of $8 \pi$ and, since $\bar{\lambda}>0$, we conclude that $\bar{\lambda}$ has to be at least $8 \pi$. On the other hand, we have $\bar{\lambda} \leqslant \lambda_{*}$ and, by Lemma4 the latter is not greater than $8 \pi$. Thus, we obtain that $\lambda_{*}$ equals $8 \pi$, finishing the proof of Theorem A. The rest of this section is devoted to the proof of Lemma 5

\subsection{Proof of Lemma 5}

To prove the lemma we analyse the structure of the nodal set of the eigenfunctions $\bar{u}_{i}$. Following the arguments of Cheng [1], this allows to bound the vanishing order of the $\bar{u}_{i}$ 's at each nodal point, and hence to estimate the dimension of $\operatorname{Span}\left(\bar{u}_{i}\right)$.

First, since the $u_{i, n}$ 's are first eigenfunctions of the Schrodinger operator $\left(-\Delta_{g}+V_{n}\right)$, then each of them changes sign. Moreover, by the results in [1] the nodal set $u_{i, n}^{-1}(0)$ 
is an immersed circle in $M$, and the complement $M \backslash u_{i, n}^{-1}(0)$ has exactly two connected components, called nodal domains. We claim that the bubble point $x$ belongs to the closure of the set

$$
\bigcup_{n} u_{i, n}^{-1}(0) \quad \text { for every } \quad i=1, \ldots, m .
$$

Indeed, for otherwise there exists a neighbourhood of $x$ which belongs to the nodal domain of $u_{i, n}$ for all sufficiently large $n$. This, in turn, implies that the limit map $\bar{u}_{i}$ does not change sign on the bubble sphere, and hence $\bar{\lambda}$ has to be a zero eigenvalue for the corresponding Schrodinger operator. The latter clearly contradicts to the fact that $\bar{\lambda}$ is positive.

A similar analysis yields that each limit eigenfunction $\bar{u}_{i}$ on the bubble sphere has exactly two nodal domains; their nodal lines are limits of renormalised arcs on the nodal lines of the $u_{i, n}$ 's. Now the structure theorem in [1] implies that any point on the nodal line $\bar{u}_{i}^{-1}(0)$ has vanishing order at most one. In more detail, the nodal set near a critical point with vanishing order $k$ is diffeomorphic to the nodal set of a spherical harmonic of order $k$ in $\mathbf{R}^{2}$, which consists of $k$ straight lines passing through the origin, see [1, Lem. 3.3]. Therefore, if $k$ is greater than one, then by [1, Lem. 3.1] the set $S^{2} \backslash \bar{u}_{i}^{-1}(0)$ has at least 3 connected components - a contradiction.

The same analysis equally applies to a non-trivial linear combination of the $\bar{u}_{i}$ 's, and we conclude that any point on its nodal line also has a vanishing order at most one. Now following [1, Th. 3.4], we show that the dimension of $\operatorname{Span}\left(\bar{u}_{i}\right), i=1, \ldots, m$, is not greater than 3. Suppose the contrary. Then for any $z \in S^{2}$ the map

$$
\operatorname{Span}\left(\bar{u}_{i}\right) \ni v \longmapsto(v(z), \nabla v(z)) \in \mathbf{R}^{3}
$$

has a non-trivial kernel - there exists a non-trivial linear combination of the $\bar{u}_{i}$ 's that vanishes at $z$ together with its first derivatives. Thus, the vanishing order at $z$ is greater than one - a contradiction.

\section{Final remarks}

1. The proof of Theorem B is based on the version of the bubbling convergence theorem for harmonic maps with a variable metric on the domain surface, see [12, Lem. 1.2]. All our arguments in Sect. 2 and 3 admit obvious adjustments to cover this case also.

2. One can analyse the concentration of extremal potentials from the point of view of the bubble tree convergence of harmonic maps, as described in [12]. (The latter is based on a different renormalisation at the bubble point than the one used in Sect. 3). More precisely, one can show that when $V_{n} \rightarrow e\left(1-\delta_{x}\right)$ the corresponding harmonic maps $U_{n}$, given by eigenfunctions, converge to a constant harmonic map with only one bubble attached at the point $x$; in other words, no "secondary" bubbles appear. Finally, mention that the equality $\bar{\lambda}=\lambda_{*}$, obtained in Sect. 3, reflects the "no energy loss at the neck" phenomenon.

3. It is extremely interesting to understand under what hypotheses analogous concentration compactness properties hold for more general (for example, maximising) sequences of potentials. This question is motivated by the existence problem for maximal (or extremal) potentials, and has strong links with isoperimetric inequalities for eigenvalues, see [10, 11].

\section{References}

[1] Cheng, S. Y. Eigenfunctions and nodal sets. Comment. Math. Helv. 51 (1976), 43-55. 
[2] Eells, J., Lemaire, L. Selected topics in harmonic maps. CBMS Regional Conference Series in Mathematics, 50. AMS, Providence, RI, 1983. v+85 pp.

[3] El Soufi, A. Ilias, S. Majoration de la seconde valeur propre d'un opérateur de Schrödinger sur une variété compacte et applications. J. Funct. Anal. 103 (1992), 294-316.

[4] El Soufi, A. Ilias, S. Riemannian manifolds admitting isometric immersions by their first eigenfunctions Pacific J. Math. 195 (2000), 91-99.

[5] Hélein, F. Harmonic maps, conservation laws and moving frames. Translated from the 1996 French original. Second edition. Cambridge Tracts in Mathematics, 150. Cambridge University Press, Cambridge, 2002. xxvi+264 pp.

[6] Hersch, J. Quatre propriétés isopérimétriques de membranes sphériques homogènes. C. R. Acad. Sci. Paris Sér. A-B 270 (1970), A1645-A1648.

[7] Jost, J. Two-dimensional geometric variational problems. Pure and Applied Mathematics. John Wiley \& Sons, Ltd., Chichester, 1991. x+236 pp.

[8] Kato, T. Perturbation theory for linear operators. Second edition. Grundlehren der Mathematischen Wissenschaften, Band 132. Springer-Verlag, Berlin-New York, 1976. xxi+619 pp.

[9] Ladyzhenskaya, O. Ural'tseva, N. Linear and quasilinear elliptic equations. Academic Press, New York-London, 1968, xviii+495 pp.

[10] Li, P., Yau, S.-T. A new conformal invariant and its applications to the Willmore conjecture and the first eigenvalue of compact surfaces. Invent. Math. 69 (1982), 269-291.

[11] Nadirashvili, N. Berger's isoperimetric problem and minimal immersions of surfaces. Geom. Funct. Anal. 6 (1996), 877-897.

[12] Parker, T. H. Bubble tree convergence for harmonic maps. J. Differential Geom. 44 (1996), 595-633.

[13] Sacks, J., Uhlenbeck, K. The existence of minimal immersions of 2-spheres. Ann. of Math. (2) 113 (1981), 1-24. 